\title{
Relevance of Visual Thinking Strategies for Psychiatry Training
}

\author{
Elizabeth Ryznar ${ }^{1}$ (D) $\cdot$ Margot Kelly-Hedrick $^{2} \cdot$ Philip Yenawine $^{3} \cdot$ Margaret S. Chisolm $^{1}$
}

Received: 11 October 2021 / Accepted: 7 January 2022 / Published online: 24 January 2022

(C) Academic Psychiatry 2022

Learning how to conduct a comprehensive examination and develop an individualized formulation for patients who present with psychiatric problems is a complex and dynamic process that takes years to master. Progression from acquisition of basic knowledge and skills to mastery requires didactic education and training as well as direct clinical practice supplemented by supervision. In addition to the process of knowledge acquisition and skills mastery that occurs during training, a parallel process of professional identity formation-in which residents internalize psychiatry's core values and beliefs - is occurring [1].

Transformational teaching conceptualizes learning as not simply the acquisition of core knowledge and skills, but also personal growth in values and beliefs [2]. A core component of transformational teaching is creating experiences that support such growth outside of the traditional learning environment [2]. A major scoping review suggests that integration of the arts and humanities into medical education has transformative potential [3].

Visual Thinking Strategies (VTS) is an arts-based pedagogic approach that - through close looking, reflection, and open discussions of visual art-fosters critical thinking skills, communication and collaboration, and personal growth [4]. VTS theory is based on how viewers experience the visual worlds; research shows that $80 \%$ of people who view art are curious about what story the art is telling [5]. Developed by Philip Yenawine and Abigail Housen for museum education, VTS has been widely used, studied, and refined over the past 30 years in a variety of educational settings with all levels of learners, from pre-K through adult, and more recently in medical learners. In multiple studies of both nonmedical and

Elizabeth Ryznar

elizabeth.ryznar.md@gmail.com

Johns Hopkins School of Medicine, Baltimore, MD, USA

Duke University School of Medicine, Durham, NC, USA

Watershed Collaborative, Baltimore, MD, USA medical learners, including randomized controlled trials, VTS has been demonstrated to promote clinically relevant skills, such as observation and critical thinking [6-11]. As an example of VTS research in medical learners, one study of a VTS workshop in first year medical students found that VTS training increased the total number of words used to describe clinical images, increased the time spent analyzing the images, and increased the number of clinically relevant observations [6]. Chisolm et al have argued elsewhere that VTS can do more than build skills; it can also promote clinical excellence [12]. To our knowledge, no reports of VTS in psychiatry training exist. Here, we describe a VTS session and explore learner and educator perspectives on its relevance to psychiatry residency training, with reference to the Accreditation Council for Graduate Medical Education (ACGME) psychiatry milestones [13].

\section{VTS Structure and Study Design}

The senior author is a certified VTS facilitator who-for the past 2 years - has implemented VTS in the psychiatry intern didactic curriculum on a quarterly basis. In a VTS session, participants observe and reflect on one work of art that has been pre-selected by the VTS facilitator $[4,5]$. The participants do not need any background or experience in art or art history, and do not receive any information about the artwork prior to the discussion. The facilitator invites participation by asking: "What's going on in this picture?" and "What do you see that makes you say that?" After one person responds, the facilitator asks, "What more can we find?" [4, 5]. The facilitator then reiterates this pattern of questions for the remainder of the session, thus encouraging deep reflection and discussion from all participants. A VTS session can range in duration from 15 to $45 \mathrm{~min}$, but a typical discussion lasts about $20 \min [4]$.

To explore formally the relevance of VTS to psychiatry residency training, the senior author assembled a team to conduct a voluntary VTS research session for psychiatry residents 
from any training year. Via in-person and email communication, we invited 47 residents to attend one 1-h VTS research session; of these, four agreed to participate (two first-year and two third-year residents). The small number of participants likely relates to timing: this occurred early during the pandemic, when we were not allowed to gather in-person and therefore could not provide food as an incentive for participating during the lunch-hour of an otherwise clinically and educationally busy day. Nonetheless, four is a sufficient number as VTS works well with groups of 4-15 participants [4, 14].

VTS cofounder Philip Yenawine facilitated a 25-min VTS session discussing a pre-selected art image (see Figure 1). The VTS session was followed by a 25-min semi-structured group interview with two central questions: "What does VTS ask of you?" and "How is this relevant to psychiatry?". Although VTS traditionally is performed in-person at an art museum, it has been adapted to a virtual format [15]. Given the nature of the COVID-19 pandemic in July 2020, we elected to hold the session via zoom.

With permission from participants, we audio-recorded the session, which was then transcribed. We qualitatively analyzed the session transcript using an interpretative phenomenological analysis. Residents provided informed consent. The study was reviewed and deemed exempt by the Johns Hopkins Medicine Institutional Review Board (IRB\# 00253553).

\section{VTS Discussion Content}

Participants described their initial reactions to the image. Two participants sensed a tenuous link from reality, commenting on the translucent figure and the line going into his head as well as on the angle of the structure on the wall. Another participant described the image as "upsetting," evoking a sense of depression or anxiety, based on the empty room,

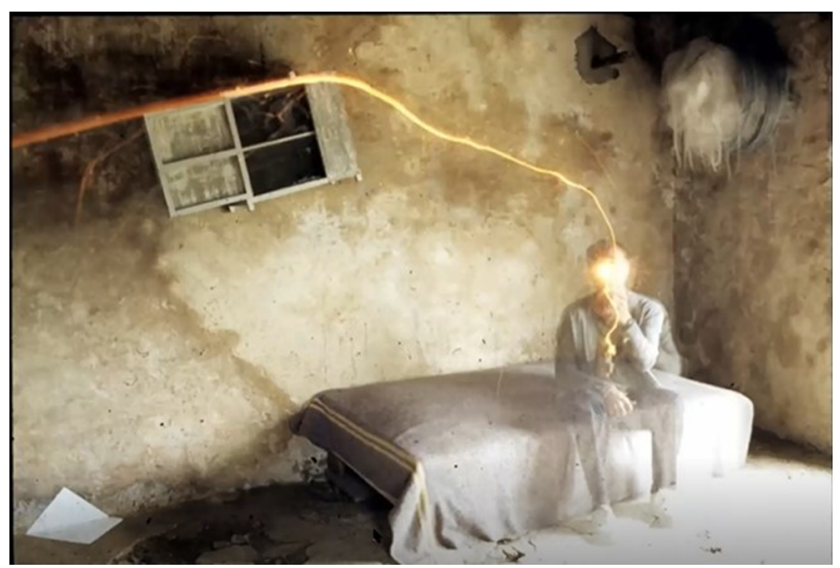

Figure 1 Andre Martheleur. Self-portrait, 1983. Photograph. Reprinted with permission of the Martheleur estate dirty-appearing structure on the wall, and shadow coming from the nearly translucent figure.

Participants also described the object on the wall in the image. One participant believed it to be a window, which added to their sense of confusion. When a fellow participant described the object as a cabinet instead, the other participant remarked how that differing characterization changed their interpretation of the image:

Because at first I thought it was a window and it would be really strange to have a window in that setting. It' $d$ be kind of melting. But once she said it was a cabinet I saw it has legs. There's a depth to it. I was like "yes, of course it's a cabinet!" It made the room seem more real and concrete and less dreamlike. So that really changed my perspective on the context.

The participants also discussed the nature of the image. One participant called it a painting, and when asked "what did you see that made you call it a painting," responded "I guess I just assumed it was a painting. I don't know why. [laughing]" Another participant then suggested it was a photograph (which it actually is), providing evidence from the image, such as the double positioning of the figure as if there were two exposures and using a long shutter time to create light paths in the image.

When asked "What more can you find?" toward the end of the discussion, participants drew their attention to other aspects of the photograph, such as the disruption in the walls and floors and the vague element in the corner of the room.

\section{Resident Perspectives on VTS}

Participants described how the prolonged analysis of this one image increased their appreciation of it, especially because the initial discomfort would have led them to quickly pass by the work if they saw it in a museum. The participants also reported that VTS required assessment of their internal state of mind, allowing them to listen to their emotions and ground them in the painting through questioning, listening, and paraphrasing. It also required a level of vulnerability in the group.

When asked about the implications of VTS for psychiatry, participants drew parallels between VTS and psychiatry residency training - having to pay attention to who is in front of you, even if it may be uncomfortable; taking the time to fully appreciate the details and context; and recognizing inherent subjectivity and how that may relate to diagnosis and treatment of a patient:

I think that psychiatrists refine their practices and who they want to treat. As residents we are exposed to all kinds of patients ... being forced to sit with something 
that's disconcerting or aren't comfortable with, may be a parallel. We are always taught that understanding a patient's context helps us understand and appreciate them. We have the same privilege when we sit with our patients for two hours and can discover who they are.

We all looked at the painting and see things differently. We all have different responses to patients and this can impact how we diagnose or treat the patients.

\section{Educator Perspectives on VTS}

VTS can provide a non-clinical setting in which to practice close observation, respect for others' - sometimes differingperspectives, and appreciation/empathy. These are essential for all physicians, but especially for psychiatry, and have implications for teaching psychiatric evaluation, diagnosis, and formulation, as well as interdisciplinary teamwork and psychotherapy.

Achieving mastery in the first ACGME patient-care milestone ("psychiatric evaluation") involves the ability to "elicit and observe subtle and unusual findings" [13]. By asking participants "What do you see that makes you say that?" VTS encourages them to ground their observations or interpretations in visual evidence. This was seen when a resident assumed the image to be a painting when it was, in fact, a complex photograph. Furthermore, by asking participants "What more can we find?" VTS encourages them to be curious and to dig deeper. These observational skills can translate to the psychiatric evaluation, where residents learn to observe appearance, behavior, speech, affect, and thinking in order to support a formulation.

The practice of basing an assessment on observed findings also has implications for psychiatric formulation and psychotherapy training (which correspond to the second and fourth patient-care milestones [13]). Grounding a diagnostic interpretation in the evidence from a comprehensive history and detailed mental status examination is crucial to accurate psychiatric formulation [16]. Grounding an interpretation in evidence is also critical in psychotherapy - trainees should not be making interpretations and psychodynamic theories freely but instead should focus specifically on what the patient is saying or doing [17].

The collaborative nature of VTS, where participants' various perspectives are shared and appreciated, also helps condition residents for collaborative teamwork and may help residents with the second interpersonal and communication skills milestone ("interprofessional and team communication") [13]. In VTS, participants communicate their impressions and listen respectfully to other participants' impressions. Often, participants will develop a new understanding together based on a triangulation of ideas, as noted by the resident quoted in the previous section. Furthermore, it is not unusual for one piece of information to change the overall impression (or diagnosis), as the participants experienced when discussing whether the structure on the wall in the image was a window or a cabinet. Thus, dealing with and integrating differing opinions in the VTS context may prepare residents to operate effectively in teams. This is very important in inpatient psychiatry and also in many forms of outpatient care, where the psychiatrist, as head of a multidisciplinary team, must integrate data and assessments from oneself, the patient, other multidisciplinary team members (social workers, psychologists, occupational therapists, nurses, case managers), and collateral informants like family and friends. The experience of listening to a broad range of responses to the same image can also be a helpful way to introduce trainees to projective tests such as the Rorschach and thematic apperception test.

Recognizing and managing emotions in oneself and others is a central task of psychiatry. Art has sometimes been viewed as a "third thing," which allows for safe exploration of emotionally complex responses outside of the direct physicianpatient relationship [18]. In choosing works of art that can come off as more unpleasant or unusual, facilitators can thus utilize VTS as a way of helping participants to sit with their discomfort and process it, as revealed in the participants' discussion above. This ability corresponds to high level mastery of another patient care milestone ("psychiatric formulation and differential diagnosis"), which requires the ability to "[integrate] clinician's and patient's emotional responses into the diagnosis and formulation." Furthermore, close observation of the artwork can also help participants assess and recognize the emotions of the figure in the image, similar to how a psychiatrist needs to do the same for the patient in front of them, a basic requirement of empathy.

Just as there are many different ways of discussing artwhether that be identification of structures and figures, or discussion of color palette and shading — so too are there many ways of formulating a patient. Trainees are taught the biopsychosocial model, the Perspectives of Psychiatry, and Diagnostic and Statistical Manual of Mental Disorders, as well as the multiple approaches within different schools of psychotherapy. VTS, with its iterative set of questions, encourages participants to look closely and find new ways of assessing the same image in front of them and thus provides a nonclinical setting in which to practice the mental flexibility required for another high-level competency within the second patient care milestone ("Psychiatric Formulation and Differential Diagnosis"): "Develops formulations based on multiple conceptual models" [13]. 


\section{Conclusions}

Arts and humanities-based teaching can provide an opportunity to practice skills, gain personal insight, appreciate multiple perspectives, and consider the culture of psychiatry and medicine $[19,20]$. In our study, psychiatry resident and educator perspectives support these functions. VTS can provide a non-clinical environment in which to practice grounding their theories in observation, exploring their emotional responses, approaching a situation from multiple lenses, and considering the unique position psychiatrists hold. These functions correspond to the various milestones identified as critical for psychiatric training [13] and indeed are critical for training astute and empathic psychiatrists. Interested readers can learn more about VTS by contacting the authors, visiting the training website [5], and/or reading additional articles [4, 14].

Funding Dr. Chisolm is the director of the Paul McHugh Program for Human Flourishing, through which her work is supported.

\section{Declarations}

Disclosures Dr. Chisolm is an Editorial Board Member for Academic Psychiatry. Manuscripts that are authored by a member of the Editorial Board undergo the same editorial review process applied to all manuscripts, including anonymous review.

Ethics Approval This study was reviewed by the Johns Hopkins University School of Medicine Institutional Review Board and deemed exempt (IRB00253553) on 7/7/2020.

\section{References}

1. Kalet A, Buckvar-Keltz L, Harnik V, Monson V, Hubbard S, Crowe R, Song HS, Yingling S. Measuring professional identity formation early in medical school. Med Teach. 2017;39(3):255-61.

2. Slavich GM, Zimbardo PZ. Transformational teaching: theoretical underpinnings, basic principles, and core methods. Educational Psychology Review. 2012;24:569-608.

3. Moniz T, Golafshani M, Gaspar CM, Adams NE, Haidet P, Sukhera J, Volpe RL, de Boer C, Lingard L. How are the arts and humanities used in medical education? Results of a scoping review. Acad Med. 2021;96(8):1213-22.
4. Kelly-Hedrick M, Chugh N, Zahra FS, Stephens M, Chisolm M. Art museum-based teaching: visual thinking strategies. Acad Med. 2022. https://doi.org/10.1097/ACM.0000000000004600.

5. Visual Thinking Strategies. [September 3, 2021]; Available from: https://vtshome.org/.

6. Agarwal GG, McNulty M, Santiago KM, Torrents H, CabanMartinez AJ. Impact of visual thinking strategies (VTS) on the analysis of clinical images: a pre-post study of VTS in first-year medical students. J Med Humanit. 2020;41(4):561-72.

7. Moorman M, Hensel D, Decker KA, Busby K. Learning outcomes with visual thinking strategies in nursing education. Nurse Educ Today. 2017;51:127-9.

8. Mitzova-Vladinov G, Torrents H. The fine art of health care: visual thinking strategies for interprofessional skills development in graduate nurse anesthesia education. Creat Nurs. 2020;26(3):e56-62.

9. Bentwich ME, Gilbey P. More than visual literacy: art and the enhancement of tolerance for ambiguity and empathy. BMC Med Educ. 2017;17(1):200.

10. Klugman CM, Peel J, Beckmann-Mendez D. Art Rounds: teaching interprofessional students visual thinking strategies at one school. Acad Med. 2011;86(10):1266-71.

11. Housen AC. Aesthetic thought, critical thinking and transfer. Arts and Learning Research. 2002;18(1):2001-2.

12. Chisolm MS, Kelly-Hedrick M, Wright SM. How visual arts-based education can promote clinical excellence. Acad Med. 2021;96(8): $1100-4$.

13. ACGME. Psychiatry Milestones. The Accreditation Council for Graduate Medical Education; 2020 [cited 2021 June 25]; 2nd Revision:[Available from: https://www.acgme.org/Portals/0/PDFs/ Milestones/PsychiatryMilestones.pdf.

14. Chisolm MS, Kelly-Hedrick M, Stephens MB, Zahra FS. Transformative learning in the art museum: a methods review. Fam Med. 2020;52(10):736-40.

15. Kelly-Hedrick M, Stouffer K, Kagan H, Yenawine P, Benskin E, Wolffe S, et al. The online art museum. MedEdPublish. 2020, 9(1).

16. Lyketsos CG, Chisolm MS. The trap of meaning: a public health tragedy. JAMA. 2009;302(4):432-3.

17. Slavney PR. Psychotherapy : an introduction for psychiatry residents and other mental health trainees. Baltimore: The Johns Hopkins University Press; 2005.

18. Gaufberg E, Batalden M. The third thing in medical education. The Clinical Teacher. 2007:4:78-81.

19. Moniz T, Golafshani M, Gaspar CM, Adams NE, Haidet P, Sukhera J, Volpe RL, de Boer C, Lingard L. The prism model: advancing a theory of practice for arts and humanities in medical education. Perspect Med Educ. 2021;10(4):207-14.

20. Moniz T, Golafshani M, Gaspar CM, Adams NE, Haidet P, Sukhera J, Volpe RL, de Boer C, Lingard L. The prism model for integrating the arts and humanities into medical education. Acad Med. 2021;96(8):1225.

Publisher's Note Springer Nature remains neutral with regard to jurisdictional claims in published maps and institutional affiliations. 